\title{
Radio Resource Sharing Framework for Cooperative Multi-operator Networks with Dynamic Overflow Modelling
}

\author{
Raouf Abozariba*, Md Asaduzzaman and Mohammad N. Patwary \\ \{r.abozariba\}, \{md.asaduzzaman\},\{m.n.patwary\}@staffs.ac.uk \\ Faculty of Computing, Engineering and Sciences (FCES), \\ Staffordshire University, Stoke-on-Trent, Staffordshire ST4 2DE, United Kingdom
}

\begin{abstract}
Due to the exponentially growing wireless services and application demand as well as the heterogeneity of the same, wireless network operators are expected to be seeking for radio resource co-operation strategies to the user group with the highest possible quality of experience (QoE). In this paper we have proposed an analytical framework for dynamic spectrum access (DSA) to adopt such cooperation within intra-network as well as inter-network operators scenarios, while sharing radio resources; assuming radio resource sharing agreement is in place. The proposed model focused onto reducing global blocking probability within a given geographical area to attain wireless services as a trade-off with increased blocking probability within local (individual network operator specific) network blocking probability; yet lower than the acceptable threshold. We derived the global balance equation and found an explicit expression of the blocking probability for each resource sharing model presented in this paper. The robustness of the proposed analytical framework is evaluated under three application specific scenarios considering various traffic intensity on demand as well as a set of global reserved resources (within one of the application specific scenarios). The results show that within a geographical area, the blocking probabilities can be reduced up to $60 \%$ with the proposed DSA framework in comparison to the existing local spectrum access schemes.
\end{abstract}

Index Terms-Communications Society, IEEE, IEEEtran, journal, IAT $_{\mathbf{E}} \mathbf{X}$, paper, template.

\section{INTRODUCTION}

Today's wireless networks are characterised by a fixed spectrum assignment policy. As a result of increasing demands for wireless applications there is a lack of frequency resources. In response, recent years has seen a significant interest in quantitative measurements of licensed and unlicensed spectrum use. Several research groups, companies and regulatory bodies have conducted studies of varying times and locations with the aim to capture the overall utilisation rate of spectrum. These studies have given a notable amount of insight on spectrum use [1, 2]. Most of these studies have shown that a large amount of allocated spectrum are under-utilised, resulting in a waste of valuable frequency resources, so-called spectrum holes [3-6].

\footnotetext{
*Corresponding author: Raouf Abozariba, Staffordshire University, College Road, Stoke-on-Trent, Staffordshire ST4 2DE, United Kingdom. Mob: +447429234875, Email: r.abozariba@staffs.ac.uk.
}

Subsequently, high blockage probabilities are imminent for many users due to the shortages of frequency resources.

Most of the current radio spectrum resources distributions are based on the static spectrum allocation principles [7], which has been identified as a major concern of spectrum scarcity. Efficient spectrum sharing was considered as one of the promising approaches to enhance the networks' Grade of Service (GoS). In order to satisfy the requirements of increased demands of wireless applications and to improve spectrum utilisation, dynamic spectrum access (DSA), along with other technologies, such as spectrum aggregations, are proposed in the literature to solve these current spectrum inefficiency problems [3, 8-12].

Providing a satisfactory GoS to users is important for the network operators. This can enable network operators to evaluate the performance degradation which they can tolerate while operating in DSA mode. The GoS improvements can also entice network operators in legacy systems to adopt DSA technology [13].

To legalise spectrum sharing, a number of spectrum regulators such as the Federal Communications Commission (FCC), approved the use of unlicensed devices in a number of licensed bands under restricted conditions [5]. As a result, innovative techniques that can offer exploiting the available spectrum under these restricted conditions are needed. However, the legalising process remains to be limited in certain geographical areas and certain frequency bands.

Resource allocation of DSA is broadly categorised by the roles of primary networks, known as the passive and active primary network models. The passive model assumes that a primary network is unaware of the operations of secondary networks (secondary networks perform spectrum sensing to determine idle spectrum for opportunistic use), and it does not require any modification for the primary network systems. However, the passive model is considered to have high complexity due to added tasks such as spectrum sensing and control overhead. In contrast, spectrum sensing in the active model is not required by secondary networks because it is assumed that a cooperation between network operators exists, and information about the frequency allocation, occupancy characteristics of the channels and other parameters can be 
exchanged. In addition, DSA allows networks, which are engaged in the cooperation, to benefit economically by leasing their respective unused spectrum resources to each other.

In conventional networks channels are allocated to users based on application requirements and spectrum availability. This allocation also depends on internal and/or external policies of the operators. It has been noted that, if innovative sharing mechanisms have to be employed, it has to be decided on a case-by-case basis [2].

In this paper we analyse spectrum sharing specific mechanisms by introducing overflow modelling, where operators are able to acquire portions of spectrum bandwidths from adjacent network operators. We focus on the analytical robustness during the interaction between network operators and on investigating their potential benefits from it. The results presented show the effective and appropriate use of DSA within the most suitable sharing scenario, in particular, bidirectional dynamic overflow model (Model 1). Our findings can be summarised as follows:

- We show that a Non-Sharing Model leads to a poor performance in terms of blocking probability as a single performance measurement, whereas there are possibilities utilise the unused spectrum within adjacent network operators.

- Dynamic overflow modelling provides a solution to gain access to the under-utilised frequency bands by using additional spectrum from adjacent operators within a given geographical region. Overflow modelling can be beneficial to the network operators, even if it comes with certain regulatory and operational limits.

- A network with dynamic and real time overflow capabilities can improve the system performance even for limited overflow such as in the uni-directional overflow model.

- Overflow mechanism in DSA is effective for reducing the overall blocking probability of the network; maximal reduction of blocking probability can be attained with a pre-agreed reserved resources among the operators within a given geographical area.

The remainder of the paper is organised as follows. Related work is presented in Section II. The detailed description of the system model is given in Section III. The proposed dynamic resource sharing algorithm is presented in Section IV, while the scenario specific DSA mechanism with overflow models are studied in Subsections IV-A, IV-B, IV-C and IV-D. Analytical results are provided in Section $\mathrm{V}$, followed by concluding remarks in Section VI.

\section{RELATED WORK}

Resource sharing mechanisms in of multi-operators networks have been studied extensively in the context of DSA and Cognitive Radio Networks (CRNs) [14-18]. In [14], the benefits of Authorised Spectrum Access (ASA) are shown, considering different methods to optimise the network's resources, by simulating an LTE network where a Mobile Network Operator (MNO) is allowed to use the $2300 \mathrm{MHz}$ band as an ASA licensee. The authors of [15] study a spectrum sharing problem in an unlicensed band where multiple systems coexist and interfere with each other. A cognitive radio system based on scheduling technology was modelled in [19]. The more recent study [16] proposes a control-free DSA algorithm for cognitive radio networks (CRNs).

Although intensive research has been done on resource sharing mechanisms, only a few studies addressed the blocking probability gain when considering overflow in coexisting networks [19-22]. The minimum blocking probabilities and maximum spectrum utilisations of three co-located systems with different bandwidth requirements were derived for onechannel band scenario in [21]. In [22], call arrivals (demand) from primary users and secondary users in the opportunistic spectrum sharing system are modelled by a Markovian arrival process which captures correlation in the aggregate arrival process consisting of the two types of call arrivals. A Markov chain analysis for spectrum access in licensed bands for cognitive radios is presented and forced termination probability, blocking probability and traffic throughput are derived in [23].

In [24], the authors focused on performance modelling for heterogeneous wireless networks based on a hierarchical overlay infrastructure. In particular, the new traffic blocked in a network due to capacity limit can be overflowed to the networks with available capacity at the higher tiers. Such traffic overflow is considered a uni-directional overflow. While in [25], the authors considered a speed-sensitive call admission control (CAC) scheme to assign overflowed calls to appropriate tiers. If the new calls of fast-speed users in a low tier network are blocked due to capacity limits, the blocked new calls are overflowed to a high-tier network for possible service. If the blocked new calls are from slow-speed users in a high tier network, they are overflowed to a low-tier network. Blocked calls from fast-speed users are overflowed to the higher tier networks with larger coverage and blocked calls from slowspeed users are overflowed to the lower tier networks with smaller coverage. A bi-directional call overflows are supported in the hierarchical heterogeneous overlay systems. In [26], a load sharing scheme was considered, an incoming voice call is preferably distributed to the cell, and overflows to the WLAN only if there is not sufficient free bandwidth for a voice call in the cell. Dynamic transfer of ongoing voice calls in the WLAN to the cell via vertical handoff whenever the cell has free bandwidth to accommodate more voice calls. Meta information of data calls that can be passed to the network layer is exploited. This scheme is also considered a bi-directional overflow model.

Five overflow policies were discussed in [27], the approach taken is to allow the new calls and handovers to compete on a first-come, first-served basis. The authors developed an analytical method that treats overflow in a unified manner. The aim was to allow the approximate performance of overflow strategies that balance the need to maintain calls in progress with the desire to accept more new calls to be evaluated for large networks.

The models discussed in the aforementioned literature are specific to hierarchal admission, type of service and mobility of users. We, on the other hand, present a detailed comparisons between various possible models for DSA, in particular we show that for a given operator the blocking probability does 
not always improve and it depends on the level of interaction between operators. Moreover, our analytical models have been derived specifically to allow for more general analysis which is crucial for the new emerging DSA applications (e.g., cognitive radio technology) and future generation of wireless telecommunications. Our investigation was conducted in order to gain a better understanding of the behaviour of the DSA networks with regard to GoS.

Resource sharing problem increases as we deal with multioperator networks. With this level of complexity, spectrum allocation has to deal with demands from a mix of types of services of these networks [28]. For example, considering TDMA and OFDM based services increases the allocation complexity further. In spite of all the above-mentioned works, there is still no work that studies the overflow between coexistent network operators when different models of resource sharing are considered. In addition, our proposed models can accommodate any number of services, which are supported by the operators.

\section{System MODEL AND ASSUMPTIONS}

In the context of this investigation, we have considered an infrastructure-based wireless network architecture where the system that owns the spectrum property rights (called the primary system) willingly and actively attempts to share its spectrum with secondary systems to enhance the global spectrum utilisation within a given geographical area. We assume that the network operators own spectrum property rights of bandwidths (contiguous and/or non-contiguous) in order to supply different kinds of services. In this context, we further assume that network operators can act both as primary or secondary systems, depending on whether they lease or borrow spectrum bandwidths, respectively. Network operators are expected to interact with each other by acquiring or leasing spectrum bandwidths owned by coexisting network operators in the same region. Secondary systems are not expected to use the infrastructure of primary system, but only acquire the right to use the incumbent spectrum of primary networks on temporal and spatial basis.

In this system model, the operators are expected to interact with each other by adjusting their actions to enhance mutual benefits. This is carried out by employing the best possible strategy for secondary and primary system with a given set of constraints to control their blocking probabilities. As shown in Figure 1, a given geographical area is covered with radio signals by a set of network operators. The operators are working in an overlapped manner to provide their respective users with a preset number of services.

We assume that each network operator supports a number of services. We denote the services as

$$
\mathbf{n}=\left(\begin{array}{cccc}
n_{1}^{1} & n_{1}^{2} & \ldots & n_{1}^{M_{1}} \\
n_{2}^{1} & n_{2}^{2} & \ldots & n_{2}^{M_{2}} \\
\vdots & \vdots & \ddots & \vdots \\
n_{N}^{1} & n_{N}^{2} & \ldots & n_{N}^{M_{N}}
\end{array}\right)
$$

where $n_{i}^{j}$ corresponds to the type of services of the $i$ th operator, $i=1,2, \ldots, N, j=1,2, \ldots, M_{i}$.
TABLE I: Symbols Used for the Analytical Modelling

\begin{tabular}{ll}
\hline \hline & \\
Notations & Descriptions \\
\hline$N$ & Number of network operators in the network \\
$M_{i}$ & Types of services at the $i$ th operator \\
$n_{i}^{j}$ & $j$ th number of service at the $i$ th operator \\
$W_{i}$ & Allocated bandwidth of the $i$ th operator \\
$S_{i}^{k}$ & Services supported by the $i$ th operator \\
$L_{i}$ & Total number of service supported by the $i$ th operator \\
$\mathcal{A}_{i}$ & Set of the available services for $i$ th operator \\
$K_{i}$ & Total number of services available for $i$ th operator \\
$n_{i}$ & Number of channel requests in progress at $i$ th operator \\
$P\left(b_{i}\right)$ & Blocking probability at $i$ th operator \\
$\lambda_{i}$ & Arrival rate at $i$ th operator \\
$\mu_{i}$ & Services Rate at $i$ th operator \\
$c_{i}$ & Capacity at the $i$ th operator \\
$X_{i}(t)$ & Number of channels required in $i$ th operator at time $t$ \\
$\Omega$ & State space \\
$\mathbf{I}_{i}$ and $\mathbf{I}_{i j}$ & Unit vector \\
$\pi(\mathbf{n})$ & Steady State \\
\hline
\end{tabular}

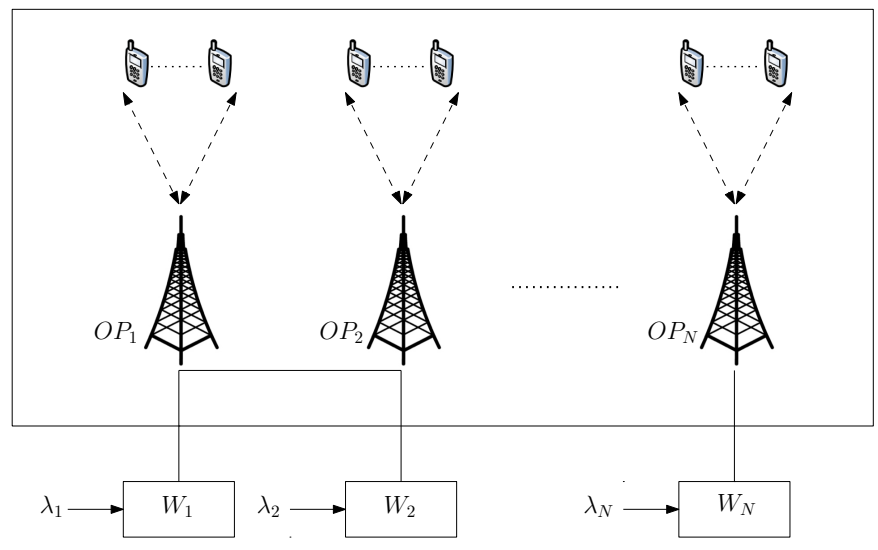

Fig. 1: System Model: Coexisting Network Operators in a DSA

Each service supported by the network is realised by a particular data rate, which are only supportive of particular operating bands such as 791-821 MHz, 880-915 MHz, and 1920-1980 MHz. Each $n_{i}^{j}$ has a capacity $c_{i}^{j}, i=1,2, \ldots, N$, $j=1,2, \ldots, M_{i}$. Hence, the capacity matrix can be written as follows.

$$
\mathbf{C}=\left(\begin{array}{cccc}
c_{1}^{1} & c_{1}^{2} & \ldots & c_{1}^{M_{1}} \\
c_{2}^{1} & c_{2}^{2} & \ldots & c_{2}^{M_{2}} \\
\vdots & \vdots & \ddots & \vdots \\
c_{N}^{1} & c_{N}^{2} & \ldots & c_{N}^{M_{N}}
\end{array}\right)
$$

We assume that the network operators consider a loss model, where there are no waiting places in the system, and block the arriving channel requests when all servers are busy [29]. Unlike the queueing type models, loss models are stable and the closed form analytical solution of blocking probability exists irrespective of traffic intensity. However, no closed form solution exists for infinite buffer queueing models if traffic intensity is greater than one, that is, if arrival rate is greater than departure rate. 
Although multiple network operators are serving in the same geographical area, due to the variation of the service provision options among networks, there may exist a variation of services which feature specific peak time slots. Subsequently, the overall spectrum utilisation may vary from one operator to another at certain intervals. This may lead the network operators into a situation when one operator experience high demand while the resources of other coexisting operators in the region are under-utilised. This means overloaded operators may utilise the underloaded spectrum resources of adjacent operators. In this paper we present an analytical framework to enhance the overall GoS among the network operators within the given geographical area. Such GoS enhancement is achieved by cooperative resource sharing between network operators in the form of dynamic traffic overflow modelling.

In the proposed overflow framework, a set of classification of operators are introduced on the basis of their cooperation agreements and traffic handling scenarios. Let us assume there are two types of network operators: the first one is willing to share resources when they are under-utilised, and the second one is unwilling to cooperate with other operators. The first type can be further divided into primary and secondary operators. Overflow from the secondary operator to the primary operator formulate a uni-directional overflow model. In the case where the same network operator can act both as primary and secondary, then such traffic handling scenarios formulate a bi-directional overflow model. Moreover, in this paper we also consider a bi-directional overflow model with reserved capacity where additional capacity is accessible for operators. For analytical tractability, only one operator in the network is considered to have access to the reserved capacity.

The overflow mechanisms and the interactions between networks operators come with the expense of more communication overhead. Information about the extent of spatial region for spectrum use and maximum power, need to be exchanged between involved operators in order to avoid interference, and as a consequence, higher exchange of information will introduce more overhead. Moreover, the realisation of the models presented in this paper may require new technologies in the form of coordination, signalling protocols, network elements and client devices which will entail additional computational power. Measurements and analysis of such communication and computation overheads would be of great value, but is beyond the scope of this paper.

\section{A. Formulation of Agreements}

One assumption in this paper is that the network operators involved in the cooperation are in some form of agreement to share their resources as predicted in the future generations of cellular networks [30,31]. The nature of such resource sharing agreements depends on several factors: primarily, on the willingness of the network operators according to their resource availability and business policy. The agreements may specify key elements in spectrum sharing, e.g., the secondary demand and payments in a given period. Cooperation provide more control over trade-offs between GoS provision and pricing. Examples of such spectrum sharing agreements which may be motivated by compensations to the spectrum owner in the form of monetary, are found in [32-35].

The level of cooperation and terms of agreements can have many forms depending on the policy of the operators. More specifically, overflow can be initiated from an Operator $i$ to Operator $j$ when the blocking probability at the Operator $i$ is

$$
P\left(b_{i}\right) \geq \epsilon_{i},
$$

where $\epsilon_{i}$ is a very small blocking probability "threshold" of the Operator $i$ and $P\left(b_{i}\right)$ is the current blocking probability of Operator $i$.

Under an agreement, Operator $j$ receives some monetary benefit for leasing channels to the Operator $i$. The amount of reward that Operator $j$ will receive from the Operator $i$ can be written in the mathematical form given by

$$
r_{i j}(t)=r_{0 j}+f\left(P\left(b_{j}^{*}\right)(t)-P\left(b_{j}\right)(t), r_{i j}^{*}(t)\right),
$$

subject to

$$
P\left(b_{j}^{*}\right)<\epsilon_{j},
$$

where $r_{0 j} \geq 0$ is a fixed reward received by the Operator $j$ due to the agreement, $P\left(b_{j}\right)(t)$ is the blocking probability of Operator $j$ due to its own arrivals at time $t, P\left(b_{j}^{*}\right)(t)$ is the new blocking probability of Operator $j$ as a result of its own arrivals as well as the overflow from Operator $i$ at time $t, r_{i j}^{*}(t)$ is the reward received from Operator $i$ due to the admission of a unit arrival to Operator $j$ at time $t$ and $\epsilon_{j}$ is the blocking probability threshold for the Operator $j$. In the event where $P\left(b_{j}^{*}\right)=\epsilon_{j}$, operator $j$ could decide to block any further overflow from operator $i$. Obviously in this case, operator $j$ will not suffer from any further performance degradation. The monetary compensation $r_{i j}(t)$ is proportional to the performance degradation incurred by overflow form operator $i$ to operator $j$. In this form of agreement, both operators may have incentives to participate in spectrum sharing: either to improve the performance, represented in reducing the blocking probability, or increase in revenues at the expense of marginal performance degradation. In this agreement, Operator $i$ charges higher rate $r_{i j}(t)$ as $P\left(b_{j}^{*}\right) \rightarrow \epsilon_{j}$. Note that a more realistic approach is when Operator $i$ considers modifying the reward according to the benefit gained by overflow, such that equation (2) can be written as

$$
r_{i j}(t)=r_{0 j}+f\left(\alpha_{i}(t), P\left(b_{i}^{*}\right)(t), P\left(b_{j}^{*}\right)(t)-P\left(b_{j}\right)(t), r_{i j}^{*}(t)\right),
$$

subject to

$$
P\left(b_{j}^{*}\right)<\epsilon_{j},
$$

and

$$
r_{i j}(t)<\alpha_{i}(t)
$$

where $\alpha_{i}(t)$ is the revenue due to overflow from the Operator $i$ to Operator $j$ at time $t$ and $P\left(b_{i}^{*}\right)(t)$ is the blocking probability of the Operator $i$ at time $t$. Such agreements are dynamic in nature and they change at each time slot $t$ as a function of the demands and rewards paid to Operator $j$. The best sharing agreement can not be determined without analysing the blocking probabilities for each network individually. To focus on the impact of spectrum sharing on the blocking 
probabilities, in the next section, we present four possible scenarios with different overflow mechanisms.

\section{Proposed Dynamic Resource Sharing ALGORITHM}

A predefined level of GoS is essential for network operators when designing or upgrading a cellular network. It constitutes one of the incentives for network operators to participate in spectrum sharing. As the number of users increase, the network operators are required to provide the users with a fixed radio resources. One way to maintain such predefined $\mathrm{GoS}$, is cooperation among network operators in the form of dynamic resource sharing. There are two fundamental aims of such dynamic resource sharing;

- Enhanced network wide GoS with efficient spectrum utilisation.

- Additional revenue generation by negotiated dynamic sub-contraction of under-utilised spectrum within each network operator.

Algorithm 1 describes a generic service selection which is used by operator $i$ to select the accessible service, where $\mathcal{A}$ is the total number of accessible services in the network, known to every operator in advance. In this service selection algorithm, an operator continues to use its allocated resources for as long as the arrival rate is lower than the capacity of the operator (e.g., $\lambda_{i}<c_{i}$ ). We will show in this Section that the Algorithm 1 ensures that if operator $i$ experience high traffic demand, the blocking probability is increases, and thus the operator $i$ can overflow to the available spectrum of adjacent operator(s), subject to accessibility and availability.

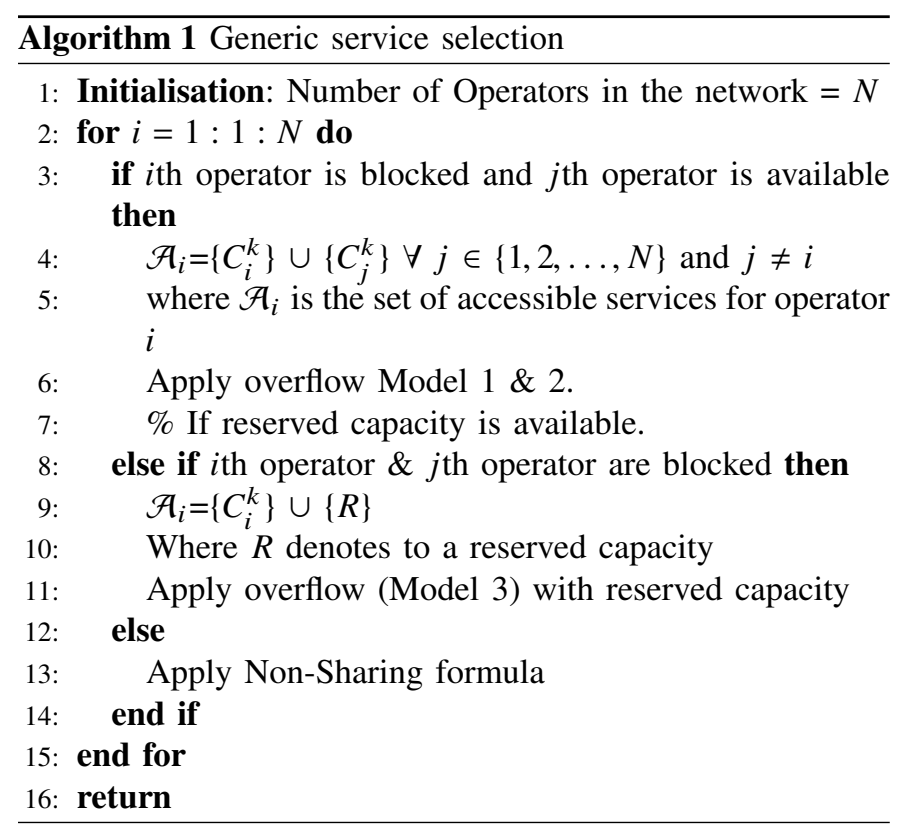

To study the proposed algorithm, we have developed four different models based on a loss system with overflow and evaluated and compared each of these models through numerical analysis.

\section{A. Non-Sharing Model}

Consider a network consisting of two operators for a cellular communications network. We assume that the two operators are in an agreement to share the spectrum if they can both support the same services. However in this model, we consider a case where there are no services in common in order for the operators to deploy resource sharing. Hence, we denote to this model as a Non-Sharing Model. A state of this network is a vector $\mathbf{n}=\left(n_{1}, n_{2}\right)$, where $n_{i}$ is the number of channel requests in progress in $i$ th operator. The topology of the network is depicted in Figure 2.

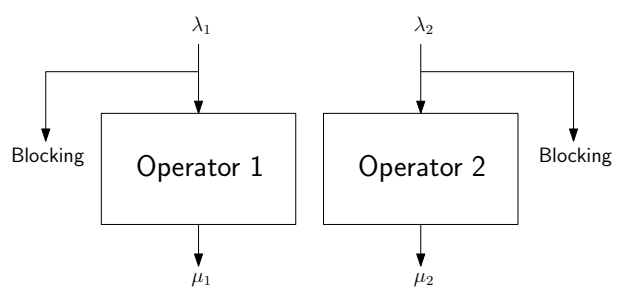

Fig. 2: Non-Sharing network with two operators

Let $\lambda_{1}$ and $\lambda_{2}$ be the arrival rates to the Operator 1 and 2 respectively, and the service rates be $\mu_{1}$ and $\mu_{2}$ and capacity $c_{1}$ and $c_{2}$, where both inter-arrival and service times are exponentially distributed random variables (r.v.). The blocking probability at the $i$ th operator $(i=1,2)$ for such an Erlang loss network can be calculated by

$$
P\left(b_{i}\right)=\frac{1}{c_{i} !}\left(\frac{\lambda_{i}}{\mu_{i}}\right)^{c_{i}}\left[\sum_{n_{i}=0}^{c_{i}} \frac{1}{n_{i} !}\left(\frac{\lambda_{i}}{\mu_{i}}\right)^{n_{i}}\right]^{-1} .
$$

The blocking probability $P\left(b_{i}\right)$ is defined as the probability that an arrival of user at operator $i$ is blocked because the capacity is saturated.

\section{B. Sharing Model 1 (Uni-directional overflow)}

We now consider a network with two operators with capacity $c_{1}$ and $c_{2}$ for Operator 1 and Operator 2, respectively. As assumed for the Non-Sharing Model, (discussed in subsection IV-A), here we assume that the two operators are in an agreement to share the spectrum if they can both support the same service. However, in this model, we consider a case where only Operator 1 can have access to the resources of Operator 2, while Operator 2 is not allowed to overflow to Operator 1 resources. Channel requests for Operator 1 and 2 follow Poisson processes with rate $\lambda_{1}$ and $\lambda_{2}$ for Operator 1 and 2, respectively, i.e. inter-arrival times are exponentially distributed random variables (r.v.). The service rate at Operator 1 (Operator 2) is exponentially distributed with mean $\mu_{1}^{-1}$ (respectively $\mu_{2}^{-1}$ ). If all $c_{1}$ capacity are occupied at Operator 1 , a channel request arriving at Operator 1 is overflowed to Operator 2 if there is an empty capacity, and blocked otherwise. Our goal is to minimise the proportion of blocked channel requests for each operator. Figure 3 shows a detailed flow of channel requests for such a network.

Let $X_{1}(t)$ be the number of channels required in Operator 1 and $X_{2}(t)$ in Operator 2 at time $t$. Also $X_{12}(t)$ denotes 


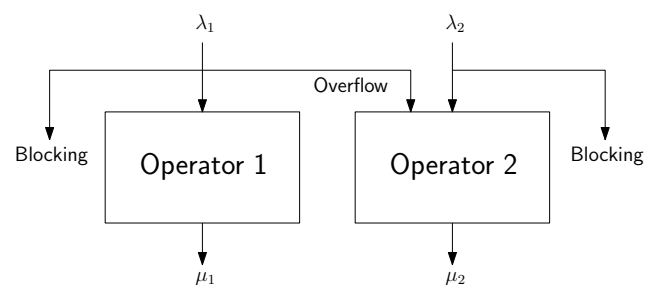

Fig. 3: A two-operator network with uni-directional overflow (Model 1)

the number of channels required in Operator 2 overflowed from Operator 1 at time $t$. The assumption of exponential distribution enables us to model the network as a continuoustime Markov chain $\mathbf{X}=\left(X_{1}(t), X_{12}(t), X_{2}(t), t \geq 0\right)$ with state space given by

$$
\Omega=\left\{\mathbf{n}=\left(n_{1}, n_{12}, n_{2}\right): n_{1} \leq c_{1}, n_{2}+n_{12} \leq c_{2}\right\},
$$

where $n_{i}, i=1,2$, is the number of channels required at the $i$ th operator and $n_{12}$ is the number of channels required at Operator 2 overflowed from Operator 1 . The transition rates $\mathbf{Q}=\left(q\left(\mathbf{n}, \mathbf{n}^{\prime}\right), \mathbf{n}, \mathbf{n}^{\prime} \in \Omega\right)$ are given by

$q\left(\mathbf{n}, \mathbf{n}^{\prime}\right)= \begin{cases}\lambda_{1} & \mathbf{n}^{\prime}=\mathbf{n}+\mathbf{I}_{1} \text { or } \quad \mathbf{n}^{\prime}=\mathbf{n}+\mathbf{I}_{12}, \text { if } n_{1}=c_{1} \\ \lambda_{2} & \mathbf{n}^{\prime}=\mathbf{n}+\mathbf{I}_{2} \\ n_{i} \mu_{i} & \mathbf{n}^{\prime}=\mathbf{n}-\mathbf{I}_{i}, \quad i=1,2 \\ n_{12} \mu_{1} & \mathbf{n}^{\prime}=\mathbf{n}-\mathbf{I}_{12} \\ 0 & \text { otherwise, }\end{cases}$

where $\mathbf{I}_{i}$ and $\mathbf{I}_{12}$ denote $i$ th unit vectors. We are interested in deriving the blocking probability, i.e. the probability that a new channel request finds all capacities are occupied in both operators 1 and 2 .

Let $\pi(\mathbf{n})=\lim _{t \rightarrow \infty} P(\mathbf{X}(t)=\mathbf{n})$ denote the equilibrium distribution that there are $\mathbf{n}$ channel requests in progress in both operators. This equilibrium distribution of $\mathbf{X}$ is the unique distribution $\pi(\mathbf{n}), \mathbf{n} \in \Omega$ that satisfies the global balance equation as shown in (10), where $\mathbf{1}_{\{\cdot\}}$ denotes the indicator function of the event or set of $\{\cdot\}$. We now derive the detailed balance equations from the global balance equation (10),

$$
\lambda_{i}\left(\pi\left(\mathbf{n}-\mathbf{I}_{i}\right)+\pi\left(\mathbf{n}-\mathbf{I}_{12}\right)\right)=\left(n_{i} \mu_{i}+n_{12} \mu_{1}\right) \cdot \pi(\mathbf{n})
$$

Equation (11) has an explicit solution which is given by

$$
\pi(\mathbf{n})=K^{-1} \frac{\left(\lambda_{1} / \mu_{1}\right)^{\left(n_{1}+n_{12}\right)}\left(\lambda_{2} / \mu_{2}\right)^{n_{2}}}{\left(n_{1}+n_{12}\right) ! n_{2} !}, \quad \forall \mathbf{n} \in \Omega
$$

and

$$
K=\sum_{\mathbf{n} \in \Omega} \frac{\left(\lambda_{1} / \mu_{1}\right)^{\left(n_{1}+n_{12}\right)}\left(\lambda_{2} / \mu_{2}\right)^{n_{2}}}{\left(n_{1}+n_{12}\right) ! n_{2} !}
$$

This equilibrium distribution is a truncated multidimensional Poisson distribution from where blocking probability can be derived. The blocking probability for operator $i, i=1,2$, is then given by

$$
\begin{aligned}
P\left(b_{i}\right)= & \sum_{\mathbf{n} \in T_{i}} \pi(\mathbf{n}) \\
= & \sum_{\mathbf{n} \in T_{i}} \frac{\left(\lambda_{1} / \mu_{1}\right)^{\left(n_{1}+n_{12}\right)}\left(\lambda_{2} / \mu_{2}\right)^{n_{2}}}{\left(n_{1}+n_{12}\right) ! n_{2} !} \\
& \cdot\left[\sum_{\mathbf{n} \in \Omega} \frac{\left(\lambda_{1} / \mu_{1}\right)^{\left(n_{1}+n_{12}\right)}\left(\lambda_{2} / \mu_{2}\right)^{n_{2}}}{\left(n_{1}+n_{12}\right) ! n_{2} !}\right]^{-1},
\end{aligned}
$$

where

$$
T_{1}=\left\{\mathbf{n} \in \Omega \mid\left(n_{1}=c_{1} \cap n_{12}+n_{2}=c_{2}\right)\right\},
$$

and

$$
T_{2}=\left\{\mathbf{n} \in \Omega \mid\left(n_{12}+n_{2}=c_{2}\right)\right\} .
$$

\section{Sharing Model 2 (Bi-directional overflow)}

We shall now extend Sharing Model 1 by adding an overflow strategy from Operator 2 to Operator 1, see Figure 4. We assume that the two operators are in an agreement to share the spectrum and both operators can support the same services. In this model, we consider a case where Operator 1 can have access to the resources of Operator 2, and likewise, Operator 2 can have access to Operator 1 resources. Hence, this model is denoted as bi-directional overflow. If all $c_{1}$ capacity are occupied at Operator 1 a channel request arriving at Operator 1 is overflowed to Operator 2 if there is an empty capacity, and blocked otherwise. Similarly a channel request arriving at Operator 2 is overflowed to Operator 1 if $c_{2}$ capacity are occupied and there is a free capacity at Operator 1 . The state space for such a process can be given by

$$
\Omega=\left\{\mathbf{n}=\left(n_{1}, n_{12}, n_{2}, n_{21}\right): n_{1}+n_{21} \leq c_{1}, n_{2}+n_{12} \leq c_{2}\right\} .
$$

Deriving the global balance equation and detailed balance equations we obtain the following solution of the steadystate distribution and the expression for blocking probability calculation for each operator

$$
\pi(\mathbf{n})=K^{-1} \frac{\left(\lambda_{1} / \mu_{1}\right)^{\left(n_{1}+n_{12}\right)}\left(\lambda_{2} / \mu_{2}\right)^{\left(n_{2}+n_{21}\right)}}{\left(n_{1}+n_{12}\right) !\left(n_{2}+n_{21}\right) !}, \quad \forall \mathbf{n} \in \Omega
$$

and

$$
K=\sum_{\mathbf{n} \in \Omega} \frac{\left(\lambda_{1} / \mu_{1}\right)^{\left(n_{1}+n_{12}\right)}\left(\lambda_{2} / \mu_{2}\right)^{\left(n_{2}+n_{21}\right)}}{\left(n_{1}+n_{12}\right) !\left(n_{2}+n_{21}\right) !} .
$$

The blocking probability can be derived from the steadystate distribution (18). The blocking probability for operator $i$, $i=1,2$, is then given by

$$
\begin{aligned}
P\left(b_{i}\right)= & \sum_{\mathbf{n} \in T_{i}} \pi(\mathbf{n}) \\
= & \sum_{\mathbf{n} \in T_{i}} \frac{\left(\lambda_{1} / \mu_{1}\right)^{\left(n_{1}+n_{12}\right)}\left(\lambda_{2} / \mu_{2}\right)^{\left(n_{2}+n_{21}\right)}}{\left(n_{1}+n_{12}\right) !\left(n_{2}+n_{21}\right) !} \\
& \cdot\left[\sum_{\mathbf{n} \in \Omega} \frac{\left(\lambda_{1} / \mu_{1}\right)^{\left(n_{1}+n_{12}\right)}\left(\lambda_{2} / \mu_{2}\right)^{\left(n_{2}+n_{21}\right)}}{\left(n_{1}+n_{12}\right) !\left(n_{2}+n_{21}\right) !}\right]^{-1},
\end{aligned}
$$

where

$$
T_{1}=\left\{\mathbf{n} \in \Omega \mid\left(n_{1}+n_{21}=c_{1} \cap n_{12}+n_{2}=c_{2}\right)\right\},
$$

and

$$
T_{2}=\left\{\mathbf{n} \in \Omega \mid\left(n_{1}+n_{21}=c_{1} \cap n_{12}+n_{2}=c_{2}\right)\right\} .
$$




$$
\begin{aligned}
{\left[\lambda _ { 1 } \left(\mathbf{1}_{\left\{n_{1}<c_{1}\right\}}(\mathbf{n})\right.\right.} & \left.\left.+\mathbf{1}_{\left\{n_{1}=c_{1}, n_{12}+n_{2}<c_{2}\right\}}(\mathbf{n})\right)+\lambda_{2}(\mathbf{n})+\sum_{i=1}^{2} n_{i} \mu_{i}+n_{12} \mu_{1}\right] \cdot \pi(\mathbf{n})=\lambda_{1}\left[\pi\left(\mathbf{n}-\mathbf{I}_{1}\right)\right. \\
& \left.+\pi\left(\mathbf{n}-\mathbf{I}_{12}\right) \mathbf{1}_{\left\{n_{1}=c_{1}, n_{12}+n_{2}<c_{2}\right\}}(\mathbf{n})\right]+\lambda_{2}\left[\pi\left(\mathbf{n}-\mathbf{I}_{2}\right)\right]+\sum_{i=1}^{2}\left(n_{i}+1\right) \mu_{i} \pi\left(\mathbf{n}+\mathbf{I}_{i}\right)+\left(n_{12}+1\right) \mu_{1} \pi\left(\mathbf{n}+\mathbf{I}_{12}\right),
\end{aligned}
$$

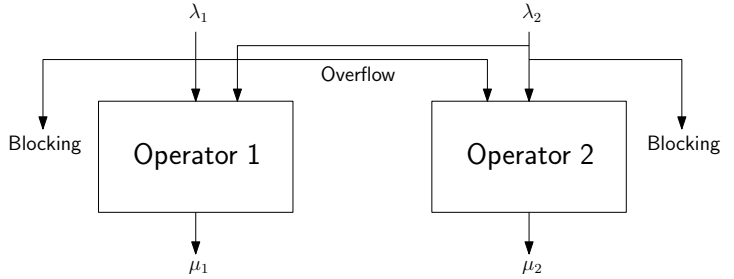

Fig. 4: A two-operator network with bi-directional overflow (Model 2)

\section{Sharing Model 3 (Bi-directional overflow with reserved capacity)}

We now consider a network consisting of two operators with bi-directional overflow from Operator 1 to Operator 2 and from Operator 2 to Operator 1 (Sharing Model 2). However, in the sharing model discussed here, we assume that there is a common spectrum pool for network operators. Each network operator is considered to posses a dedicated portion of this pooled spectrum. For analytical purposes, we only consider a case where only Operator 2 has such a dedicated spectrum portion with a defined capacity. This is to enable a certain predictable level of GoS for Operator 2. In this paper we will denote to this spectrum portion as reserved capacity. The reserved capacity can be used to reduce blocking probability at Operator 2.

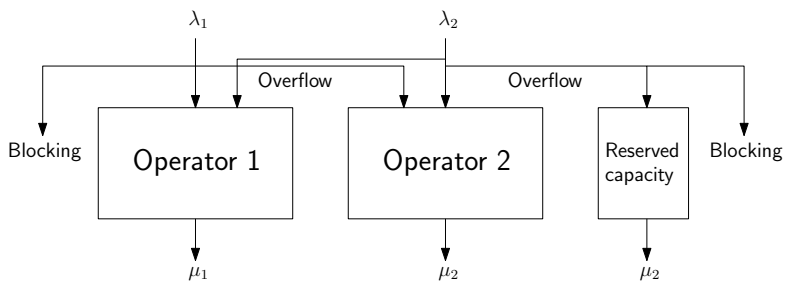

Fig. 5: A two-operators network with bi-directional overflow and reserved resources (Model 3)

Let $X_{1}(t)$ be the number of channel requests in Operator 1 and $X_{2}(t)$ in Operator 2 at time $t$. Also $X_{12}(t)$ denotes the number of channel requests in Operator 2 overflowed from Operator 1 and $X_{21}(t)$ denotes the number of channel requests in Operator 1 overflowed from Operator 2 at time $t$. Capacity at Operator 1 and 2 are denoted by $c_{1}$ and $c_{2}$ respectively. If there is no empty channel to admit a channel request in Operator 2 and in the overflow at Operator 1 then the request will be transferred to the reserved resource with capacity $c_{3}$. A state of the network can be written as $\mathbf{X}=\left(X_{1}(t), X_{12}(t), X_{2}(t), X_{21}(t), X_{23}(t), t \geq 0\right)$ with state space given by

$$
\Omega=\left\{\mathbf{n}=\left(n_{1}, n_{12}, n_{2}, n_{21}, n_{23}\right): n_{1}+n_{21} \leq c_{1},\right.
$$

$$
\left.n_{2}+n_{12} \leq c_{2}, n_{23} \leq c_{3}\right\},
$$

where $n_{i}, i=1,2$, is the number of channel requests at the $i$ th operator and $n_{i j}$ is the number of requests overflowed at operator $j$ from operator $i, i, j \in\{1,2,3\}$. The transition rates $\mathbf{Q}=\left(q\left(\mathbf{n}, \mathbf{n}^{\prime}\right), \mathbf{n}, \mathbf{n}^{\prime} \in \Omega\right)$ are given by

$q\left(\mathbf{n}, \mathbf{n}^{\prime}\right)=\left\{\begin{array}{lll}\lambda_{1} & \mathbf{n}^{\prime}=\mathbf{n}+\mathbf{I}_{1} \text { or } \mathbf{n}^{\prime}=\mathbf{n}+\mathbf{I}_{12} \text { if } n_{1}=c_{1} \\ \lambda_{2} & \mathbf{n}^{\prime}=\mathbf{n}+\mathbf{I}_{2} \text { or } \mathbf{n}^{\prime}=\mathbf{n}+\mathbf{I}_{21} \text { if } n_{2}=c_{2} \\ n_{i} \mu_{i} & \mathbf{n}^{\prime}=\mathbf{n}-\mathbf{I}_{i}, i=1,2 \\ n_{i j} \mu_{i} & \mathbf{n}^{\prime}=\mathbf{n}-\mathbf{I}_{i j}, i, j \in\{1,2\} \\ n_{23} \mu_{2} & \mathbf{n}^{\prime}=\mathbf{n}-\mathbf{I}_{23} \\ 0 & \text { otherwise. }\end{array}\right.$

The global balance equation of the system is derived and given in equation (25). The detailed balance equations obtained from the global balance equation (25) is given by

$\lambda_{i}\left(\pi\left(\mathbf{n}-\mathbf{I}_{i}\right)+\pi\left(\mathbf{n}-\mathbf{I}_{i j}-\mathbf{I}_{23}\right)\right)=\left(n_{i} \mu_{i}+n_{i j} \mu_{i}+n_{23} \mu_{2}\right) \cdot \pi(\mathbf{n})$.

The explicit solution of the detailed balance equations after normalisation $\left(\sum \pi(\mathbf{n})=1\right)$ we get

$$
\pi(\mathbf{n})=K^{-1} \frac{\left(\lambda_{1} / \mu_{1}\right)^{\left(n_{1}+n_{12}\right)}\left(\lambda_{2} / \mu_{2}\right)^{\left(n_{2}+n_{21}+n_{23}\right)}}{\left(n_{1}+n_{12}+n_{23}\right) !\left(n_{2}+n_{21}\right) !}, \quad \forall \mathbf{n} \in \Omega
$$

and

$$
K=\sum_{\mathbf{n} \in \Omega} \frac{\left(\lambda_{1} / \mu_{1}\right)^{\left(n_{1}+n_{12}\right)}\left(\lambda_{2} / \mu_{2}\right)^{\left(n_{2}+n_{21}+n_{23}\right)}}{\left(n_{1}+n_{12}\right) !\left(n_{2}+n_{21}+n_{23}\right) !}
$$

The blocking probability can be derived from the steady-state distribution (27). The blocking probability for station $i, i=$ 1,2 , is then given by

$$
\begin{aligned}
P\left(b_{i}\right)= & \sum_{\mathbf{n} \in T_{i}} \pi(\mathbf{n}) \\
= & \sum_{\mathbf{n} \in T_{i}} \frac{\left(\lambda_{1} / \mu_{1}\right)^{\left(n_{1}+n_{12}\right)}\left(\lambda_{2} / \mu_{2}\right)^{\left(n_{2}+n_{21}+n_{23}\right)}}{\left(n_{1}+n_{12}\right) !\left(n_{2}+n_{21}+n_{23}\right) !} \\
& \cdot\left[\sum_{\mathbf{n} \in \Omega} \frac{\left(\lambda_{1} / \mu_{1}\right)^{\left(n_{1}+n_{12}\right)}\left(\lambda_{2} / \mu_{2}\right)^{\left(n_{2}+n_{21}+n_{23}\right)}}{\left(n_{1}+n_{12}\right) !\left(n_{2}+n_{21}+n_{23}\right) !}\right]^{-1},
\end{aligned}
$$

where

$$
T_{1}=\left\{\mathbf{n} \in \Omega \mid\left(n_{1}+n_{21}=c_{1} \cap n_{12}+n_{2}=c_{2}\right)\right\},
$$

and

$$
T_{2}=\left\{\mathbf{n} \in \Omega \mid\left(n_{1}+n_{21}=c_{1} \cap n_{12}+n_{2}=c_{2} \cap n_{23}=c_{3}\right)\right\} .
$$

The models discussed in this paper can be summarised by Figure 6. Even though the models discussed in this paper only consider the interactions between two operators, it can be extended to include more operators with added complexity. 


$$
\begin{aligned}
{\left[\lambda _ { 1 } \left(\mathbf{1}_{\left\{n_{1}+n_{21}<c_{1}\right\}}\right.\right.} & \left.+\mathbf{1}_{\left\{n_{1}+n_{21}=c_{1}, n_{12}+n_{2}<c_{2}\right\}}\right)+\lambda_{2}\left(\mathbf{1}_{\left\{n_{21}+n_{2}<c_{2}\right\}}+\mathbf{1}_{\left\{n_{12}+n_{2}=c_{2}, n_{1}+n_{21}<c_{1}\right\}}+\mathbf{1}_{\left\{n_{12}+n_{2}=c_{2}, n_{1}+n_{21}=c_{1}, n_{23}<c_{3}\right\}}\right) \\
& \left.+\sum_{i=1}^{2} n_{i} \mu_{i}+\sum_{i, j \in\{1,2\}} n_{i j} \mu_{i}+n_{23} \mu_{2}\right] \cdot \pi(\mathbf{n})=\lambda_{1}\left[\pi\left(\mathbf{n}-\mathbf{I}_{1}\right)+\pi\left(\mathbf{n}-\mathbf{I}_{12}\right) \mathbf{1}_{\left\{n_{1}+n_{21}=c_{1}, n_{12}+n_{2}<c_{2}\right\}}\right]+\lambda_{2}\left[\pi\left(\mathbf{n}-\mathbf{I}_{2}\right)\right. \\
& \left.+\pi\left(\mathbf{n}-\mathbf{I}_{21}\right) \mathbf{1}_{\left\{n_{12}+n_{2}=c_{2}, n_{21}+n_{2}<c_{2}\right\}}+\pi\left(\mathbf{n}-\mathbf{I}_{23}\right) \mathbf{1}_{\left\{n_{12}+n_{2}=c_{2}, n_{21}+n_{2}=c_{2}, n_{23}<c_{3}\right\}}\right] \\
& +\sum_{i=1}^{2}\left(n_{i}+1\right) \mu_{i} \pi\left(\mathbf{n}+\mathbf{I}_{i}\right)+\sum_{i, j \in\{1,2\}}\left(n_{i j}+1\right) \mu_{i} \pi\left(\mathbf{n}+\mathbf{I}_{i j}\right)+\left(n_{23}+1\right) \mu_{2} \pi\left(\mathbf{n}+\mathbf{I}_{23}\right)
\end{aligned}
$$

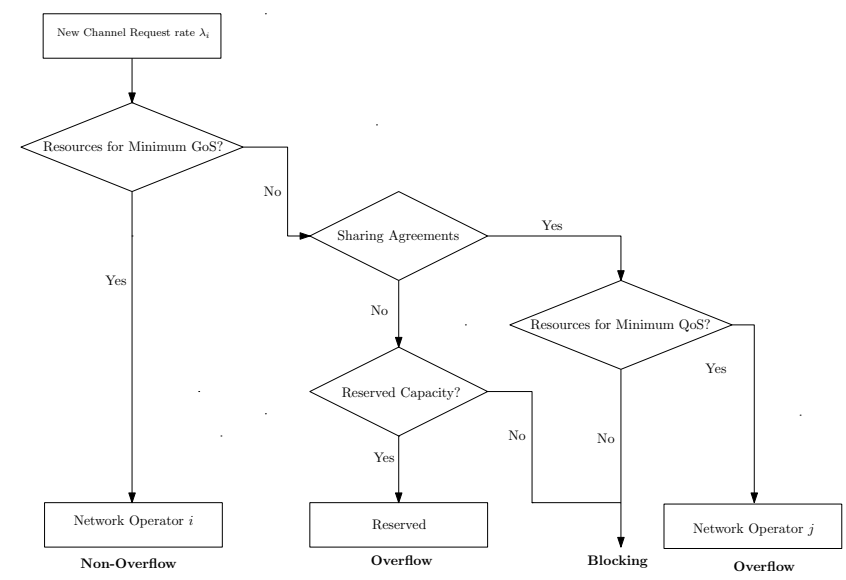

Fig. 6: The flowchart for the proposed overflow models

\section{AnALysis And Results}

In this section we investigate the robustness of the analytical models which are discussed in Section IV, "Proposed Dynamic Resource Sharing Algorithm", with different offered load (0$30)$, number of server $(0-25)$ and reserved capacity $(0,1)$ across the network. The performance of the proposed resource sharing framework is examined. For the analytical results, it is reasonable that we compare the four scenario specific model configurations: Non-Sharing Model, Sharing Model 1, Sharing Model 2 and Sharing Model 3.

\section{A. Performance comparison between Non-Sharing Model and Model 1}

The comparison for Non-Sharing Model and the proposed uni-directional overflow model at Operator 1 and Operator 2 are presented in Figure $7 \mathrm{a}$ and $7 \mathrm{~b}$, respectively. The offered load at Operator 1 varies from 0 to 30 while the offered load at Operator 2 is kept fixed at 10. Figure 7a shows the blocking probabilities for the Non-Sharing Model and the proposed uni-directional overflow model. According to the analytical results in Figure $7 \mathrm{a}$, it is clear that the blocking probability for the proposed overflow model for Operator 1 is reduced in comparison to the Non-Sharing Model. However, for the overflow model, the blocking probability for both Operator 1 and 2 converges as $\lambda_{1} \rightarrow 30$. This is due to the fact that the uni-directional sharing model only allows overflow from Operator 1 to Operator 2. Thus, the capacity for both operators reach saturation gradually as the offered load increases. In addition, for the same offered load in Non-Sharing Model and uni-directional overflow model, it is seen that at Operator 1 with our proposed overflow model when $\lambda_{1}>10$, the blocking probability is lower than those for Non-Sharing Model. This shows superiority of our proposed model. To realise the impact of our overflow model on Operator 2 with different offered load values, we have fixed the offered load at Operator 1 as 10 and varied it for Operator 2 from 0 to 30 , see Figure $7 \mathrm{~b}$. The blocking probability of Operator 2 is higher for Model 1 , except for when $\lambda_{2}<10$, because the overflow load from Operator 1. In addition the blocking probability for Operator 1 has decreased as compared to Operator 1 when employing the Non-Sharing Model. It is evident from Figure 7b that the blocking probability for the uni-directional model at Operator 1 is lower than those for Non-Sharing Model. However, in the proposed model, the blocking probability increase with the increase of offered load. This is due to the reason that as $\lambda_{2} \rightarrow 30$, the capacity gain obtained from sharing decrease with the decrease of the capacity of Operator 2. In terms of performance under different number of server, we have compared the blocking probability for Non-Sharing Model with uni-directional overflow model where the number of server at Operator 1 varies from 5 to 25 . The number of server was fixed at 10 for Operator 2. For simplicity, in this configuration, we set $\lambda_{1}=\lambda_{2}=10$. According to the analytical results, see Figure $8 \mathrm{a}$, the blocking probability at Operator 1 for our proposed model is lower than that for Non-Sharing Model. However, as $c_{1} \rightarrow 25$, the superiority over the Non-Sharing Model becomes less visible due to the fact that Operator 1 increases its own capacity. Thus, it becomes less dependant on Operator 2, which result in lower overflow levels. In addition, it is also noticed that the blocking probability for Operator 2 with both models are almost the same when the number of server exceed 10. In order to test the impact of varying the number of server at Operator 2, we kept the number of server at Operator 1 fixed at 10. For this configuration, we have fixed the offered load for Operator 1 and 2 at 10. The comparison is intended to be representative of the performance in terms of blocking probability at Operator 2 , see Figure 8a. It can be seen that as $c_{1} \rightarrow 25$, the blocking probability of Operator 1 and 2 decreases. The overflow model performs slightly better than Non-Sharing Model, while the overflow model at Operator 1 can achieve the lowest blocking probability. This analysis is used to show that a non-sharing approach where the operators do not share resources, although in some cases might perform well, does not perform well when the offered load is high. 


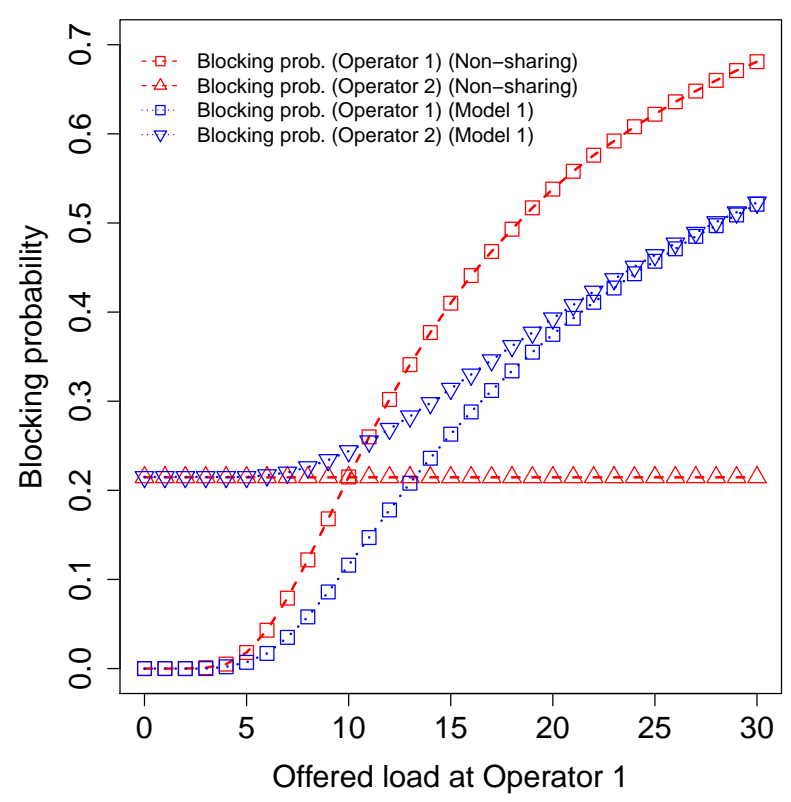

(a)

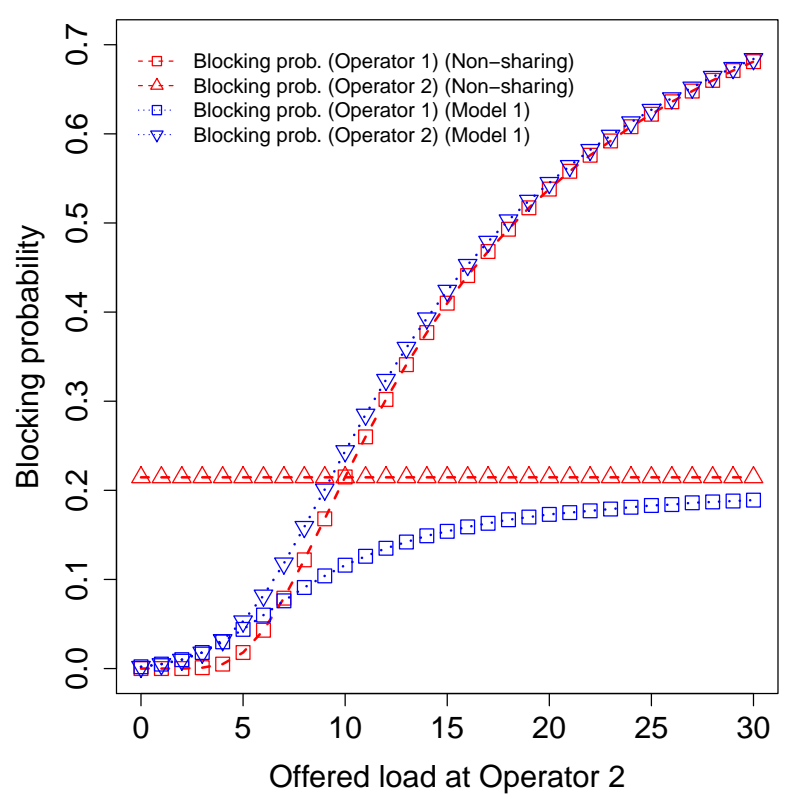

(b)

Fig. 7: Comparison of blocking probability for Non-Sharing Model with Model 1 with $c_{1}=c_{2}=10$ for (a) $\lambda_{1}=0: 30$, $\lambda_{2}=10$ and (b) $\lambda_{1}=10, \lambda_{2}=0: 30$

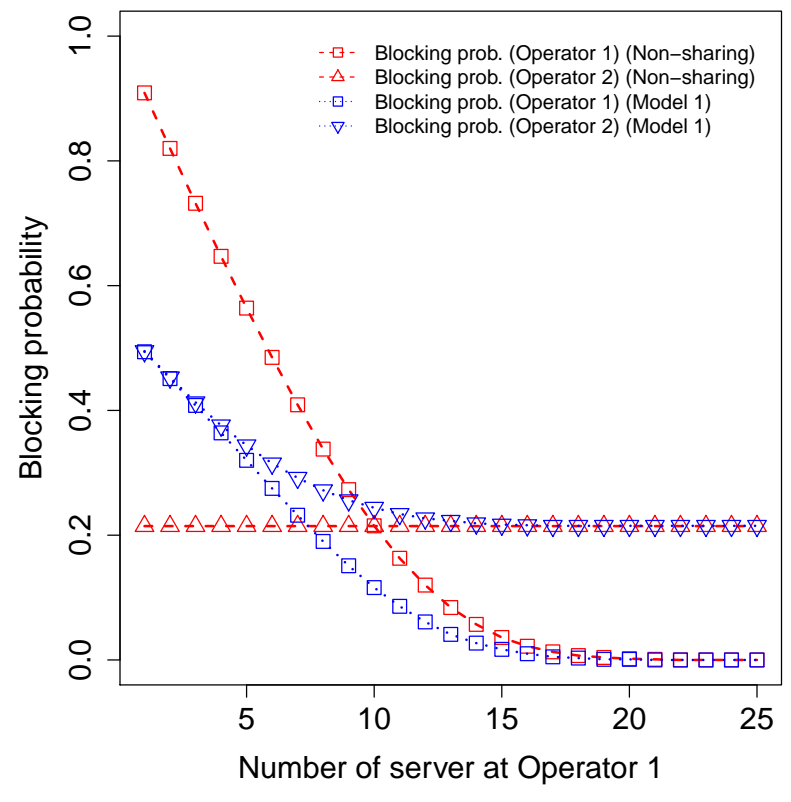

(a)

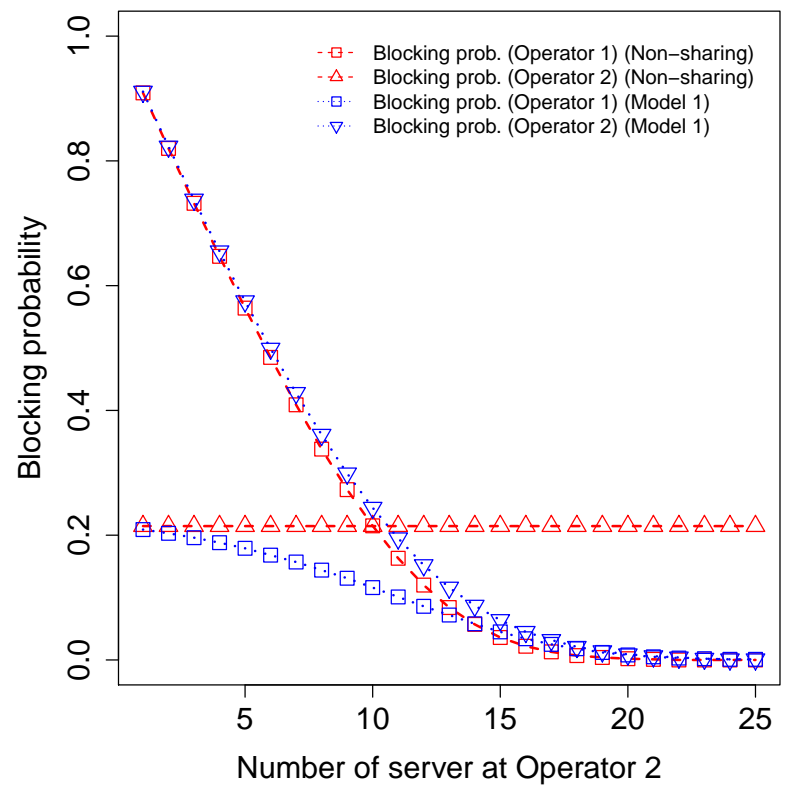

(b)

Fig. 8: Comparison of blocking probability for Non-Sharing Model with Model 1 with $\lambda_{1}=\lambda_{2}=10$ for (a) $c_{1}=5: 25$, $c_{2}=10$ and (b) $c_{1}=10, c_{2}=1: 25$ 


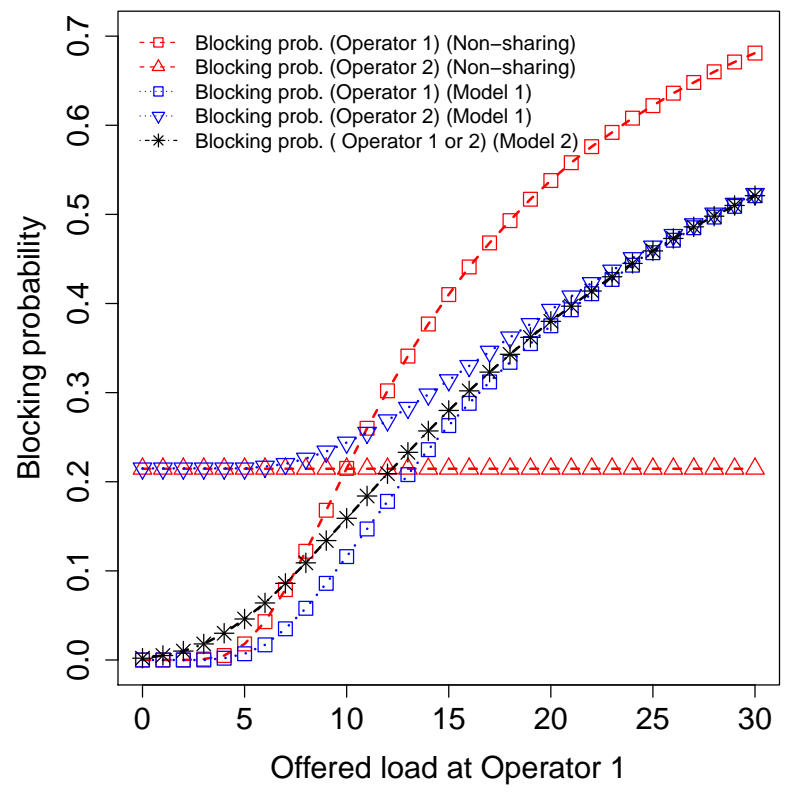

(a)

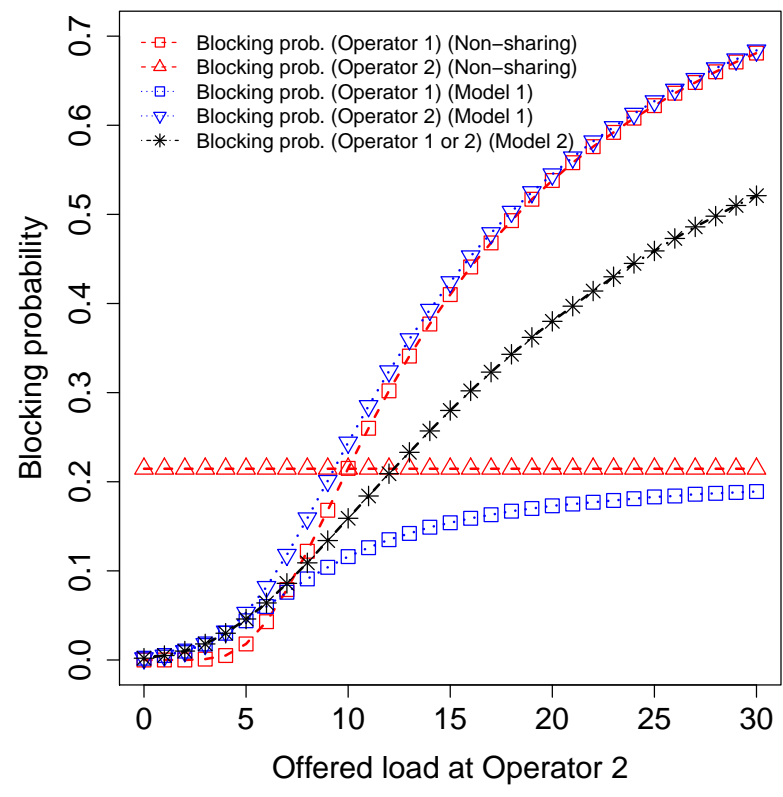

(b)

Fig. 9: Comparison of blocking probability for Non-Sharing Model with Model 1 and Model 2 with $c_{1}=c_{2}=10$ for (a) $\lambda_{1}=0: 30, \lambda_{2}=10$ and (b) $\lambda_{1}=10, \lambda_{2}=10$

\section{B. Performance comparison between Non-Sharing Model, Model 1 and Model 2}

The results obtained in Figure 9a, 9b, 10a and 10b represent a comparison of the bi-directional model with the unidirectional and Non-Sharing Model. Figure 9a demonstrates the blocking probability for the case where the offered load is varied and $c_{1}=10$. We see that the blocking probability for Operator 1, when considering Model 1, is lower than in Model 2, especially in the region where the offered load is between 5 and 15. The performance of Operator 2 in Figure $9 \mathrm{~b}$ is identical to the case in Figure 9a since the traffic load is always distributed uniformly over the two operators. The other results in Figure 10a and 10b, represent a comparison of the bi-directional model with the uni-directional and Non-Sharing Model for varying number of server. When considering individual operators it is evident from the results that Model 1 present better GoS as compared to the other two models. These results show comparisons in achieving lower blocking probability for an operator using baseline assumptions for several parameters.

\section{Performance comparison between Non-Sharing Model and Model 3}

Figure 11a and Figure 11b present the comparison of blocking probabilities for Non-Sharing Model and Model 3. Figure 11a shows the effect of increasing traffic intensity at Operator 1 , where we demonstrate that the blocking probability is lower when considering the Non-Sharing Model as compared to Model 3. The reason for this is that in Model 3 when the traffic at Operator 2 requires more capacity the set up allows for overflow to Operator 1 first rather than to the reserved capacity which is set to 5 . This creates more traffic intensity at Operator 1, which explains the observed blocking probabilities at Operator 1 in Model 3. In Figure 11b we have fixed the traffic intensity at Operator 1 while at Operator 2 the traffic is varied from $(0-30)$. In this example, at higher traffic intensity (e.g., $\lambda_{2}>5$ ) Operator 2 in Model 3 shows significant blocking probability reduction in comparison to Non-Sharing Model due to available capacity from Operator 1 as well as the reserved capacity. At low traffic intensity (e.g., $\lambda_{2}<5$ ) at Operator 1, Model 3 performs better compared to Non-Sharing Model. The number of server used for Figure 11a and Figure $11 \mathrm{~b}$ are illustrated in Table II.

The effect of number of server on blocking probability at Operator 1 and Operator 2 for Non-Sharing Model and Model 3 is presented in Figure 12a and Figure 12b, respectively. The traffic intensity is kept fixed for both operators. The results in Figure 12a shows that the blocking probability for the NonSharing Model at Operator 1 is lower than Model 3. The reason is related to the overflow of traffic from Operator 2, which adds an extra traffic at Operator 1. On the other hand, the blocking probability in the proposed Model 3 presents higher gain from the overflow flexibility, which benefits from the extra capacity provided by both Operator 1 and the reserved capacity. From Figure 11 and 12 we notice that for a particular operator, Model 3 does not always enhance the grade of service (GoS), instead the Non-Sharing Model can serve a higher GoS. The number of server used for Figure 12a and Figure 12b are illustrated in Table III. 


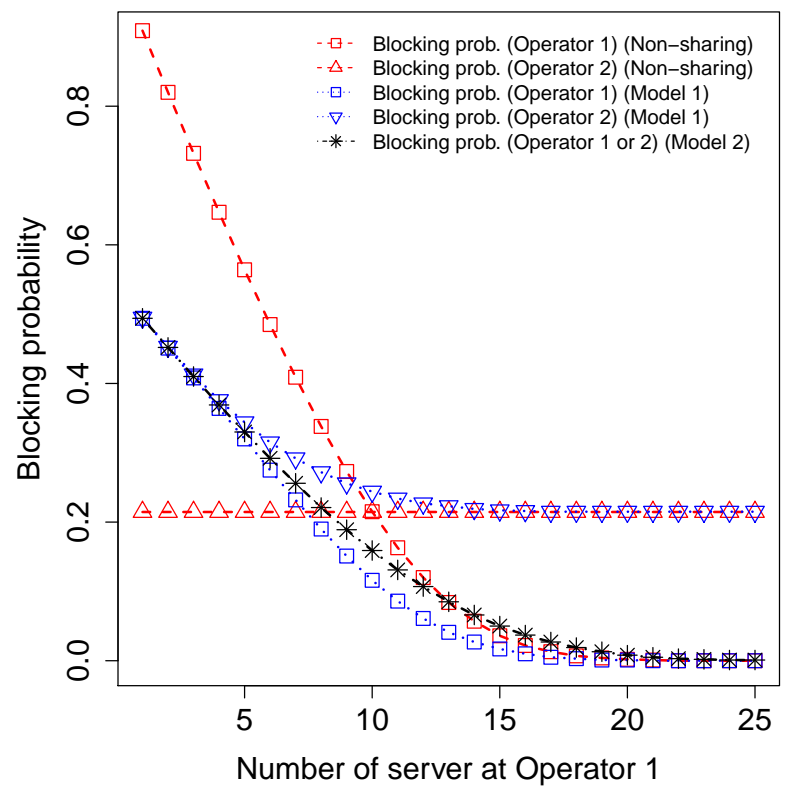

(a)

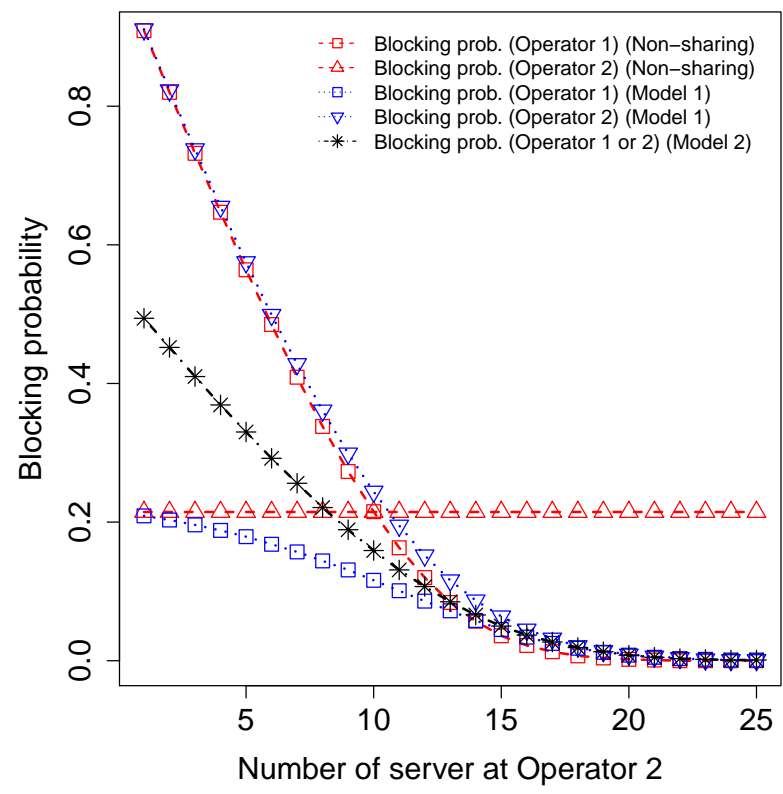

(b)

Fig. 10: Comparison of blocking probability for Non-Sharing Model with Model 1 and Model 2 with $\lambda_{1}=\lambda_{2}=10$ for (a) $c_{1}=1: 25, c_{2}=10$ and (b) $c_{1}=10, c_{2}=1: 25$

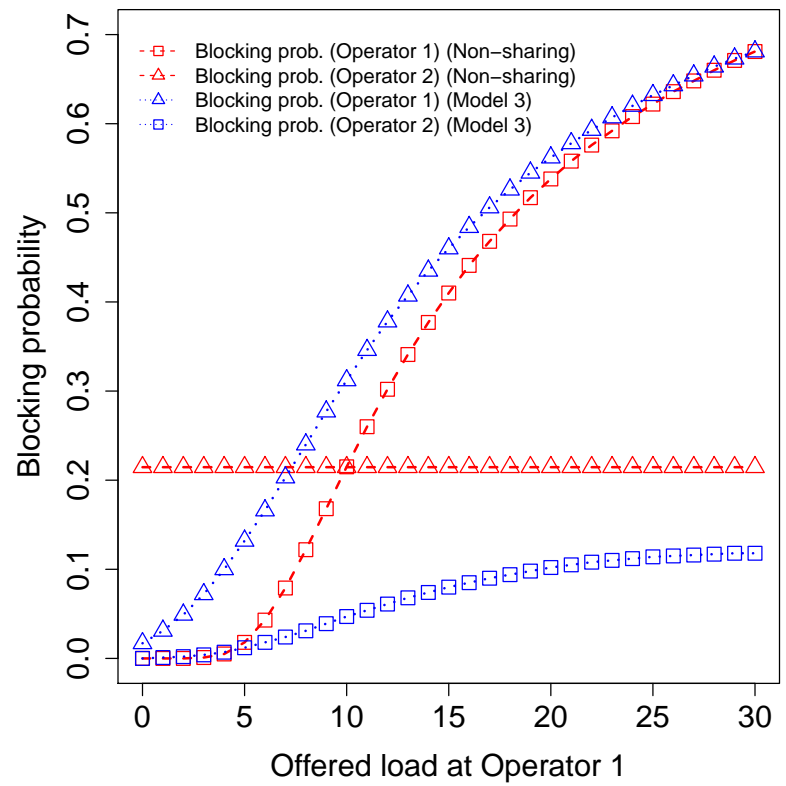

(a)

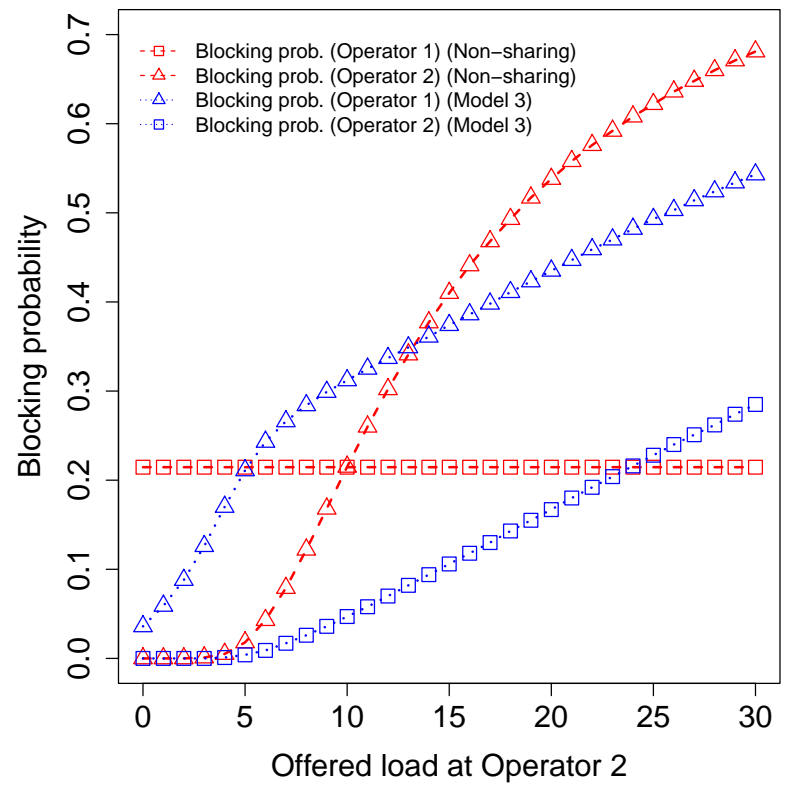

(b)

Fig. 11: Comparison of blocking probability for Non-Sharing Model $\left(c_{1}=c_{2}=10\right)$ with Model $3\left(c_{1}=10, c_{2}=5\right.$, reserved capacity $=5$ ) for (a) $\lambda_{1}=0: 30, \lambda_{2}=10$ and (b) $\lambda_{1}=10, \lambda_{2}=0: 30$

TABLE II: Number of server considered in Figure 11a and Figure 11b

\begin{tabular}{lccc|ccc}
\hline Model & \multicolumn{4}{c}{ Figure 11a } & \multicolumn{3}{c}{ Figure 11b } \\
\cline { 2 - 7 } & \multicolumn{9}{c}{ Number of server } \\
\cline { 2 - 7 } & Operator 1 & Operator 2 & Reserved & Operator 1 & Operator 2 & Reserved \\
\hline Non-Sharing & 10 & 10 & -- & 10 & 10 & -- \\
\hline Model 3 & 10 & 5 & 5 & 10 & 5 & 5 \\
\hline
\end{tabular}




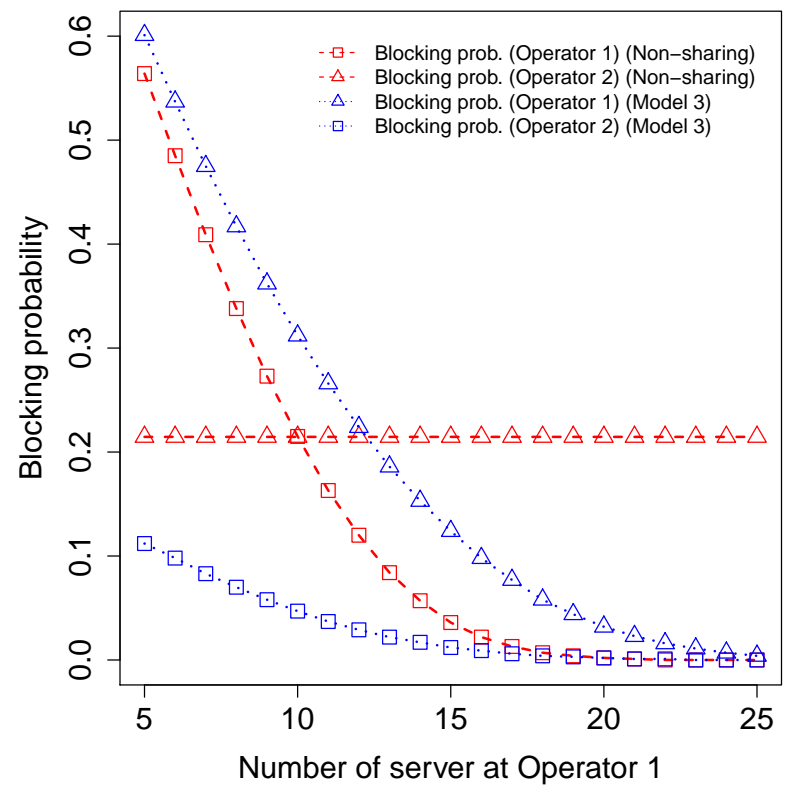

(a)

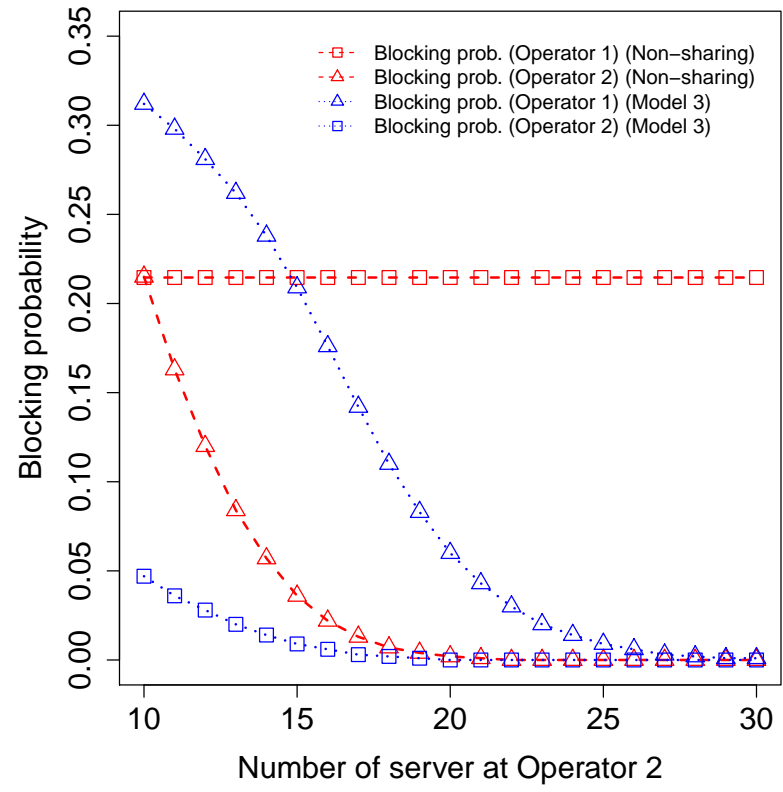

(b)

Fig. 12: Comparison of blocking probability for Non-Sharing Model with Model 3 for $\lambda_{1}=\lambda_{2}=10$. See Table III for server configurations for (a) and (b).

TABLE III: Number of server considered in Figure 12a and Figure 12b

\begin{tabular}{lccc|ccc}
\hline Model & \multicolumn{3}{c}{ Figure 11a } & \multicolumn{3}{c}{ Figure 11b } \\
\cline { 2 - 7 } & \multicolumn{5}{c}{ Number of server } \\
\cline { 2 - 7 } & Operator 1 & Operator 2 & Reserved & Operator 1 & Operator 2 & Reserved \\
\hline Non-Sharing & $5: 25$ & 10 & -- & 10 & $10: 30$ & -- \\
\hline Model 3 & $5: 25$ & 5 & 5 & 10 & $5: 25$ & 5 \\
\hline
\end{tabular}

\section{Evaluation of models under homogeneous traffic intensity}

We have compared the blocking probability for Non-Sharing Model, sharing Model 1, 2 and 3, see Table IV. The table shows the overall network blocking probability for each model configuration. Note that we defined the overall blocking probability of the networks as

$$
\begin{array}{r}
P(b)=\sum_{i=1}^{n} P\left(b_{1}\right) g\left(\lambda_{1}, \mu_{1}\right)+P\left(b_{2}\right) g\left(\lambda_{2}, \mu_{2}\right)+ \\
\ldots+P\left(b_{n}\right) g\left(\lambda_{n}, \mu_{n}\right),
\end{array}
$$

where $P\left(b_{i}\right)$ is the blocking probability at operator $i$ and $g\left(\lambda_{i}, \mu_{i}\right)$ is a function of arrival rate and service rate for the $i$ th operator, which give the weight for the $i$ th operator. In our case we assumed

$$
g\left(\lambda_{i}, \mu_{i}\right)=\frac{\lambda_{i} / \mu_{i}}{\lambda_{1} / \mu_{1}+\lambda_{2} / \mu_{2}+\ldots+\lambda_{n} / \mu_{n}} .
$$

With three different offered loads $(0.25,0.5$, and 1) at Operator 1 and 2, we calculate the blocking probability for individual operators and overall network gain. To evaluate the models under homogeneous traffic intensity, in Table IV we present a case where the four models have equal total capacity. In Model 3, $c_{1}=2$ and $c_{2}=1$, however, Operator 2 can overflow to the reserved capacity in case of no capacity is available at Operator 2 and Operator 1. Table IV shows that Model 3 has a clear advantage over Non-Sharing Model and Model 1 in terms of overall blocking probability. On the other hand, Model 3 has higher blocking probability in comparison to Model 2, this is because the overflow capacity available to Operator 1 is less in Model 3 than in Model 2 which provoke lower spectrum sharing.

\section{E. Evaluation of models under heterogeneous traffic intensity}

To better understand the models behaviour, Table $\mathrm{V}$ shows the comparison of blocking probabilities among Non-Sharing Model, sharing Model 1, 2 and 3 for heterogeneous traffic intensity. It also shows the overall network blocking probability for each model configuration. From Table V, it can be concluded that sharing Model 2 and sharing Model 3 have superiority over Non-Sharing Model and Model 1. However, if we compare Model 2 and 3 we see that Model 2 provides the lowest overall blocking probability. This indicates that even for heterogeneous traffic intensity Model 2 provides better GoS with respect to overall network performance. Since the available capacity for both operators in Model 2 is higher, the network ensures better resource utilisation as opposed to sharing Model 3. Even though the total capacity at Model 
TABLE IV: Comparison of blocking probabilities for Non-Sharing Model with Model 1, Model 2 and Model 3 with homogeneous traffic intensity

\begin{tabular}{lcccccccc}
\hline Model & \multicolumn{2}{c}{ Traffic intensity } & \multicolumn{3}{c}{ Capacity } & \multicolumn{3}{c}{ Blocking probability } \\
\cline { 2 - 9 } & Operator 1 & Operator 2 & Operator 1 & Operator 2 & Reserved & Operator 1 & Operator 2 & Overall \\
\hline Non & 0.25 & 0.25 & 2 & 2 & -- & 0.024 & 0.024 & 0.024 \\
Sharing & 0.50 & 0.50 & 2 & 2 & -- & 0.077 & 0.077 & 0.077 \\
& 1 & 1 & 2 & 2 & -- & 0.200 & 0.200 & 0.200 \\
\hline Model 1 & 0.25 & 0.25 & 2 & 2 & -- & 0.001 & 0.025 & 0.013 \\
& 0.500 & 0.500 & 2 & 2 & -- & 0.011 & 0.081 & 0.046 \\
& 1 & 1 & 2 & 2 & -- & 0.069 & 0.220 & 0.145 \\
\hline Model 2 & 0.250 & 0.250 & 2 & 2 & -- & 0.002 & 0.002 & 0.002 \\
& 0.500 & 0.500 & 2 & 2 & -- & 0.015 & 0.015 & 0.015 \\
& 1 & 1 & 2 & 2 & -- & 0.095 & 0.095 & 0.095 \\
\hline Model 3 & 0.250 & 0.250 & 2 & 1 & 1 & 0.013 & 0.001 & 0.007 \\
& 0.500 & 0.500 & 2 & 1 & 1 & 0.067 & 0.010 & 0.039 \\
& 1 & 1 & 2 & 1 & 1 & 0.225 & 0.058 & 0.142 \\
\hline
\end{tabular}

3 equal to the total capacity available to Model 2, the latter performs better due to the restriction imposed on the reserved capacity which is accessible only by Operator 1 . However, the results for blocking probability with respect to Operator 2 is the best in Model 3 due to the reserved capacity which is available only for Operator 2. In Summary, we have analysed and compared the performance of three different overflow models with Non-Sharing Model. As a result the performance achievable by the operators varies according to the operator parameters (e.g. capacity, traffic intensity) and the overflow interactions between operators. It implies that operators have the incentive to participate in the proposed sharing models since they can achieve reduced blocking probability as compared to Non-Sharing Model.

\section{CONCLUSION}

Co-operative resource sharing is considered to be one of the key challenges within future generation wireless communication networks. Considering a number of application specific scenarios, we address their issues in the paper and presented a robust analytical framework of DSA. We have studied the blocking probability of different resource sharing models under different overflow constraints. In practice, these constraints can depend on many factors such as agreements between network operators and spectrum availability. We have derived analytical models for blocking probability of network operators for the four different models: Non-Sharing Model, sharing model with uni-directional overflow (Model 1), sharing model with bi-directional overflow (Model 2) and sharing model where we consider a reserved capacity for one of the operators in the network (Operator 2) and a bi-directional overflow between both operators (Model 3). We derived the global balance equation and found an explicit expression of the blocking probability for each resource sharing model presented in this paper. The results have shown that the operators can achieve a noticeable blocking probability reduction, under the proposed models compared with the Non-Sharing Model. Our analytical results provide a basis for further study on this type of overflow with different configurations. The results highlight in general terms the importance of overflow in mobile communications. To obtain better view on the blocking probability of the networks along with the models discussed in this paper, we have calculated an overall blocking probability and summarise the robustness of the proposed framework. The analysis provided in this paper can be used to inform network operators to determine agreements terms for any future spectrum sharing cooperation with coexisting network operators.

\section{ACKNOWLEDGEMENT}

We would like to thank the Editor, Associate Editor and all the reviewers for their valuable comments and suggestions to improve the quality of the paper.

\section{REFERENCES}

[1] A. Palaios, J. Riihijarvi, P. Mahonen, V. Atanasovski, L. Gavrilovska, P. Van Wesemael, A. Dejonghe, and P. Scheele, "Two days of spectrum use in europe," in 7th International ICST Conference on Cognitive Radio Oriented Wireless Networks and Communications (CROWNCOM), 2012 pp. 24-29.

[2] M. Hoyhtya, M. Matinmikko, X. Chen, J. Hallio, J. Auranen, R. Ekman, J. Roning, J. Engelberg, J. Kalliovaara, T. Taher, A. Riaz, and D. Roberson, in 9th International Conference on Cognitive Radio Oriented Wireless Networks and Communications (CROWNCOM).

[3] I. Akyildiz, W.-Y. Lee, and K. Chowdhury, "Spectrum management in cognitive radio ad hoc networks," IEEE Network, vol. 23, no. 4, pp. 6-12, 2009.

[4] B. Wang and K. Liu, "Advances in cognitive radio networks: A survey," IEEE Journal of Selected Topics in Signal Processing, vol. 5, no. 1, pp. 5-23, Feb. 2011.

[5] P. Kolodzy, "Spectrum policy task force," Federal Communications Commission, Tech. Rep. ET Docket No. 02- 135, Nov. 2002.

[6] A. Wyglinski, M. Nekovee, and Y. Hou, Cognitive radio communications and networks: principles and practice. Academic Press, 2010.

[7] X. Liu, C. Zhang, and X. Tan, "Double-threshold cooperative detection for cognitive radio based on weighing," in 6th International ICST Conference on Communications and Networking in China (CHINACOM), 2011, pp. 205-209.

[8] I. Akyildiz, W.-Y. Lee, M. C. Vuran, and S. Mohanty, "A survey on spectrum management in cognitive radio networks," IEEE Communications Magazine, vol. 46, no. 4, pp. 40-48, Apr. 2008.

[9] P. Lin, J. Jia, Q. Zhang, and M. Hamdi, "Dynamic spectrum sharing with multiple primary and secondary users," IEEE Transactions on Vehicular Technology, vol. 60, no. 4, pp. 1756-1765, May 2011.

[10] P. Si, H. Ji, F. Yu, and V. Leung, "Optimal cooperative internetwork spectrum sharing for cognitive radio systems with spectrum pooling," IEEE Transactions on Vehicular Technology, vol. 59, no. 4, pp. 17601768, May 2010.

[11] V. Asghari and S. Aissa, "End-to-end performance of cooperative relaying in spectrum-sharing systems with quality of service requirements," IEEE Transactions on Vehicular Technology, vol. 60, no. 6, pp. 26562668, Jul. 2011. 
TABLE V: Comparison of blocking probability for Non-Sharing Model, Model 1, Model 2 and Model 3 with heterogeneous traffic intensity

\begin{tabular}{lcccccccc}
\hline Model & \multicolumn{3}{c}{ Traffic intensity } & \multicolumn{3}{c}{ Capacity } & \multicolumn{3}{c}{ Blocking probability } \\
\cline { 2 - 9 } Non & Operator 1 & Operator 2 & Operator 1 & Operator 2 & Reserved & Operator 1 & Operator 2 & Overall \\
\hline Sharing & 0.250 & 0.500 & 2 & 2 & -- & 0.024 & 0.077 & 0.059 \\
& 0.500 & 0.250 & 2 & 2 & -- & 0.077 & 0.024 & 0.059 \\
& 0.500 & 1 & 2 & 2 & -- & 0.077 & 0.200 & 0.159 \\
& 1 & 0.500 & 2 & 2 & -- & 0.200 & 0.077 & 0.159 \\
\hline Model 1 & 0.250 & 0.500 & 2 & 2 & -- & 0.003 & 0.077 & 0.052 \\
& 0.500 & 0.25 & 2 & 2 & -- & 0.006 & 0.028 & 0.013 \\
& 0.500 & 1 & 2 & 2 & -- & 0.021 & 0.204 & 0.143 \\
& 1 & 0.500 & 2 & 2 & -- & 0.043 & 0.100 & 0.062 \\
\hline Model 2 & 0.25 & 0.50 & 2 & 2 & -- & 0.006 & 0.006 & 0.006 \\
& 0.50 & 0.25 & 2 & 2 & -- & 0.006 & 0.006 & 0.006 \\
& 0.50 & 1 & 2 & 2 & -- & 0.048 & 0.048 & 0.048 \\
& 1 & 0.5 & 2 & 2 & -- & 0.048 & 0.048 & 0.048 \\
\hline Model 3 & 0.25 & 0.50 & 2 & 1 & 1 & 0.035 & 0.005 & 0.015 \\
& 0.50 & 0.25 & 2 & 1 & 1 & 0.035 & 0.002 & 0.024 \\
& 0.50 & 1 & 2 & 1 & 1 & 0.142 & 0.035 & 0.070 \\
& 1 & 0.500 & 2 & 1 & 1 & 0.143 & 0.019 & 0.101 \\
\hline
\end{tabular}

[12] T. Kwon and J. Cioffi, "Spatial spectrum sharing for heterogeneous simo networks," IEEE Transactions on Vehicular Technology, vol. 63, no. 2, pp. 688-702, Feb. 2014.

[13] A. Sahoo, M. Souryal, and M. Ranganathan, "Implementation of an opportunistic spectrum access scheme with disruption qos," in 9th International Conference on Cognitive Radio Oriented Wireless Networks and Communications (CROWNCOM), Jun. 2014, pp. 335-340.

[14] E. Perez, K.-J. Friederichs, I. Viering, and J. Diego Naranjo, "Optimization of authorised/licensed shared access resources," in 9th International Conference on Cognitive Radio Oriented Wireless Networks and Communications (CROWNCOM), Jun. 2014, pp. 241-246.

[15] R. Etkin, A. Parekh, and D. Tse, "Spectrum sharing for unlicensed bands," IEEE Journal on Selected Areas in Communications, vol. 25, no. 3, pp. 517-528, Apr. 2007.

[16] C. Xin, M. Song, L. Ma, S. Shetty, and C.-C. Shen, "Control-free dynamic spectrum access for cognitive radio networks," in IEEE International Conference on Communications (ICC), May 2010, pp. 1-5.

[17] W. Song, W. Zhuang, and Y. Cheng, "Load balancing for cellular/wlan integrated networks," IEEE Network, vol. 21, no. 1, pp. 27-33, Jan. 2007.

[18] C. Leong, W. Zhuang, Y. Cheng, and L. Wang, "Optimal resource allocation and adaptive call admission control for voice/data integrated cellular networks," IEEE Transactions on Vehicular Technology, vol. 55, no. 2, pp. 654-669, Mar. 2006.

[19] P. Zhu, J. Li, and X. Wang, "Scheduling model for cognitive radio," in 3rd International Conference on Cognitive Radio Oriented Wireless Networks and Communications (CROWNCOM), May 2008, pp. 1-6.

[20] M. Fidler and A. Rizk, "A guide to the stochastic network calculus," IEEE Communications Surveys Tutorials, vol. 17, no. 1, pp. 92-105, Firstquarter 2015.

[21] L. Luo and S. Roy, "Analysis of dynamic spectrum access with heterogeneous networks: Benefits of channel packing scheme," in IEEE Global Telecommunications Conference (GLOBECOM), Nov. 2009, pp. 1-7.

[22] S. Tang and B. Mark, "Modeling an opportunistic spectrum sharing system with a correlated arrival process," in IEEE Wireless Communications and Networking Conference (WCNC), Mar. 2008, pp. 3297-3302.

[23] X. Zhu, L. Shen, and T.-S. Yum, "Analysis of cognitive radio spectrum access with optimal channel reservation," IEEE Communications Letters, vol. 11, no. 4, pp. 304-306, Apr. 2007.

[24] Q. Huang, K.-T. Ko, and V. Iversen, "Performance modeling for heterogeneous wireless networks with multiservice overflow traffic," in Global Telecommunications Conference, 2009. (GLOBECOM 2009. IEEE), Nov. 2009, pp. 1-7.

[25] Q. Huang, Y.-C. Huang, K.-T. Ko, and V. Iversen, "Loss performance modeling for hierarchical heterogeneous wireless networks with speedsensitive call admission control," IEEE Transactions on Vehicular Technology, vol. 60, no. 5, pp. 2209-2223, Jun. 2011.

[26] W. Song and W. Zhuang, "Multi-service load sharing for resource management in the cellular/wlan integrated network," IEEE Transactions on Wireless Communications, vol. 8, no. 2, pp. 725-735, Feb. 2009.
[27] P. Fitzpatrick, C. S. Lee, and B. Warfield, "Teletraffic performance of mobile radio networks with hierarchical cells and overflow," IEEE Journal on Selected Areas in Communications, vol. 15, no. 8, pp. 15491557, Oct. 1997.

[28] M. Buddhikot and K. Ryan, "Spectrum management in coordinated dynamic spectrum access based cellular networks," in First IEEE International Symposium on New Frontiers in Dynamic Spectrum Access Networks, 2005. (DySPAN 2005), Nov. 2005, pp. 299-307.

[29] G. Giambene, Queuing theory and telecommunications. Springer, 2005.

[30] P. Ahokangas, K. Horneman, H. Posti, M. Matinmikko, T. Hanninen, S. Yrjola, and V. Goncalves, "Defining x201c; co-primary spectrum sharing x201d; x2014; a new business opportunity for mnos?" in 9th International Conference on Cognitive Radio Oriented Wireless Networks and Communications (CROWNCOM), Jun. 2014, pp. 395-400.

[31] L. Duan, J. Huang, and B. Shou, "Duopoly competition in dynamic spectrum leasing and pricing," IEEE Transactions on Mobile Computing, vol. 11, no. 11, pp. 1706-1719, 2012.

[32] C. Gizelis, D. D. Vergados, et al., "A survey of pricing schemes in wireless networks," IEEE Communications Surveys \& Tutorials, vol. 13, no. 1, pp. 126-145, 2011.

[33] L. Gao, J. Huang, Y.-J. Chen, and B. Shou, "An integrated contract and auction design for secondary spectrum trading," IEEE Journal on Selected Areas in Communications, vol. 31, no. 3, pp. 581-592, 2013.

[34] G. Kasbekar, S. Sarkar, K. Kar, P. Muthuswamy, and A. Gupta, "Dynamic contract trading in spectrum markets," IEEE Transactions on Automatic Control, vol. 59, no. 10, pp. 2856-2862, 2014.

[35] L. Gao, X. Wang, Y. Xu, and Q. Zhang, "Spectrum trading in cognitive radio networks: A contract-theoretic modeling approach," IEEE Journal on Selected Areas in Communications, vol. 29, no. 4, pp. 843-855, 2011. 